\title{
Disinformation y misinformation, posverdad y fake News Precisiones conceptuales, diferencias, similitudes y yuxtaposiciones
}

Alonso Estrada-Cuzcano

Universidad Nacional Mayor de San Marcos, Lima, Perú I mestradac@unmsm.edu.pe / https://orcid.org/oooo-0o01-5039-1108

\author{
Karen Alfaro-Mendives \\ Universidad Nacional Mayor de San Marcos, Lima, Perú |kalfarom_af@unmsm.edu.pe / https://orcid.org/oooo-0002-6218-4998 \\ Valeria Saavedra-Vásquez \\ Universidad Nacional Mayor de San Marcos, Lima, Perú | diana.saavedra@unmsm.edu.pe / https://orcid.org/oooo-0oo2-2748-2172
}

\begin{abstract}
Resumen
A través de la revisión documental, con base al enfoque cualitativo e interpretativo, el presente trabajo pretende realizar un recuento de los principales términos utilizados actualmente en el manejo de la información: disinformation, misinformation, posverdad y fakenews. Términos que evidencian la relevancia que ha adquirido la información obtenida a través de las redes sociales y que, en consecuencia, generan un desorden informativo. Esto es posible debido al crecimiento exponencial de la información en un entorno supeditado al desarrollo de las tecnologías de la información y comunicación (TIC), donde constantemente se pone en tela de juicio su veracidad y como resultado se pierde su valor y se hace cada vez más difícil la elección de información veraz y correcta. Los resultados permiten realizar precisiones conceptuales, identificar las relaciones existentes de similitud, diferencias y yuxtaposición en cada uno de los términos que han surgido en este contexto con algunos ejemplos.
\end{abstract}

Disinformation and Misinformation, Post-truth and fake news: conceptual precisions, differences, similarities and juxtapositions

\section{Abstract}

Through the documentary review, based on the qualitative and interpretative approach, the present work intends to recount the main terms currently used in information management: disinformation, misinformation, post-truth and fake news. Terms that demonstrate the relevance that the information obtained through social networks has acquired and that, consequently, generate an informative disorder.

Palabras clave

Disinformation Misinformation Posverdad Fake News

\section{Keywords}

Disinformation Misinformation Post-truth

Fake News This is possible due to the exponential growth of information in an environment 
subject to the development of information and communication technologies (ICT), where the veracity of it is constantly questioned and as a result its value is lost and it makes the choice of truthful and correct information increasingly difficult. The results allow conceptual clarifications, identify existing relationships of similarity, differences and juxtaposition in each of the terms that have emerged in this context with some examples.

Artículo recibido: 07-11-2019. Aceptado: 05-05-2020

\section{Introducción}

La problemática que da inicio al presente estudio se debe a las confusiones conceptuales sobre los términos Disinformation y Misinformation, Posverdad y Fake News, por ende, se torna necesario identificar sus similitudes, diferencias y yuxtaposiciones entre ellos analizando la literatura existente y el papel del profesional de la información actualmente, así como su rol en este contexto, considerando que la globalización, en el campo de la información, ha permitido que se manejen y difundan grandes cantidades de datos e información cotidianamente; sin embargo, los mecanismos para identificar la información falsa, sin sustento científico u objetivo, son mínimos.

Últimamente, ante la propagación de la pandemia coronavirus (COVID-19), la Organización Mundial de la Salud alertó sobre la infodemia (infodemic) como un peligro de desinformación que puede generar zozobra social. Del-Fresno-García (2019) los llama "desórdenes informativos" (desinformación, fake news, hechos alternativos, posverdad, etc.) que define como producciones intencionales cuya estrategia consiste en la fabricación de la duda y falsas controversias con el fin de conseguir beneficios económicos o ideológicos. Los desórdenes informativos están interrelacionados entre sí y dependen, de forma necesaria, de las tecnologías post internet, lo que ha modificado la naturaleza misma de la comunicación interpersonal colectiva y tienen su origen en distintas causas que han facilitado su desarrollo, alcance e impacto actual.

Morales Campos (2018) señala el constante riesgo para los usuarios y para las bibliotecas ante la desinformación, debido a la sobreexposición a los grandes volúmenes de información, la incertidumbre y la duda ante la veracidad de la información que corre por las redes o por los medios impresos, muchas veces distinta a la información que proveen las instituciones y servicios a través de ventanas públicas y de acceso abierto. El progresivo aumento de la práctica intencional de la posverdad y de las noticias falsas - que se presentan como verdad, pero sin ninguna verificación y comprobación de los datos utilizados, debido a que no se basan en hechos-facilita la manipulación de la información originada sobre bases falsas o parcialmente erróneas. Tal es así, que "cuando la información llega al usuario, le es muy difícil discriminar lo falso de lo verdadero" (Morales Campos, 2018: 105).

Desde hace mucho tiempo, en la literatura científica y la propia ficción han sugerido utopías y distopías informativas relacionadas con la satisfacción en el consumo de información. Una utopía recurrente es la referida al acceso irrestricto a la información, según las necesidades, sin limitaciones y de manera equitativa, conducentes a la denominada biblioteca total en la que el usuario puede acceder a la información y generar conocimiento desde cualquier lugar, con las herramientas y recursos adecuados (Ranganathan, 1931). 
Se denomina distopía, según la Real Academia Española (RAE, 2016), a una "representación ficticia de una sociedad futura de características negativas causantes de la alienación humana". Si se revisa la literatura ligada a las distopías informativas se puede citar a dos obras modélicas que ponen de manifiesto un futuro en donde la posibilidad de gestar la verdad y permitir la libre circulación de la información es negada, estas son las obras 1984 de Orwell y Fahrenheit 451 de Bradbury. Estos dos libros hacen referencia a distopías informativas: sociedades donde la información es manipulada, ocultada o censurada o simplemente fabrican 'mentiras verdaderas' $y$ la crítica al status quo es reprobada.

Por ejemplo, en el libro 1984, el partido de gobierno, a través del Ministerio de la Verdad, se encarga de reconstruir y/o fabricar el pasado y darles a sus ciudadanos periódicos, películas, libros, comedias y novelas con instrucciones y entretenimiento (Orwell, 2016) situación que también se desarrolla en Fahrenheit 451 que aborda el tema de la censura de libros por considerarlos peligrosos, el hombre que lee es un peligro, un obstáculo para lograr el dominio de la mente de las personas y para la felicidad; y se advierte lo siguiente:

Dale a la gente concursos que puedan ganar recordando la letra de las canciones más populares, o los nombres de las capitales de Estado, o cuánto maíz produjo Iowa el año pasado. Atibórralos de datos no combustibles, lánzales encima tantos «hechos» que se sientan abrumados, pero totalmente al día en cuanto a información. Entonces, tendrán la sensación de que piensan, tendrán la impresión de que se mueven sin moverse. Y serán felices, porque los hechos de esta naturaleza no cambian. No les des ninguna materia delicada como Filosofía o Sociología para que empiecen a atar cabos. Por ese camino se encuentra la melancolía (Bradbury, 1953: 61).

Estos futuros distópicos muestran sociedades dependientes de una tecnología avanzada, ubicadas en la Hiperhistoria, caracterizada por el dominio de lo digital y la dependencia tecnológica en la que se sumerge la sociedad y son parte de nuestra existencia, tal como lo denomina Floridi y Taddeo (2016); pero son proclives a un alto nivel de censura y además promueven una política de Estado basada en la desinformación orientadas a establecer un dominio total, aunque en la novela no se percibieron los intereses privados predominantes en la actualidad. Si bien, en el siglo XXI la libertad de opinión y de información son derechos protegidos por la legislación y los ciudadanos conocen y hacen uso de estos derechos, es importante mencionar que con la irrupción de las tecnologías de la información y comunicación (TIC) se ha incrementado el acceso a la información, pero también se incrementó la información de baja calidad y errónea, al colocar en la misma posición a las noticias reales y a las noticias falsas.

Hace algún tiempo Goldmann, en los Coloquios Royaumont, primigeniamente delineó el término 'conciencia posible' (del alemán ZugerechteBewusstsein) y señaló que "todo elemento subjetivo, aun el más valorizador o el más deformante, constituye en cuanto hecho psicosocial una realidad objetiva, e inversamente, toda comprobación, aunque sea la más rigurosa, se hace en el interior de una conciencia y, con ello, resulta ser también un hecho subjetivo" en consecuencia, la verdad o falsedad de un hecho es propia del sujeto. Por otro lado, también agregó que "todo hecho humano, individual o social, se presenta en efecto, como un esfuerzo global de adaptación de un sujeto al mundo ambiente" (Goldmann, 1966:35-36) y los mensajes que el individuo recibe, deforma, acepta o rechaza o simplemente obvia, son un reconocimiento del impacto de la posverdad o de las fakenews o noticias falsas que dejan de lado los hechos para privilegiar la distorsión de la realidad que asume el sujeto en este proceso de adaptación al mundo. 
Ante la aparición de diversos términos para referirse a la información errónea o manipulada se inicia un debate sobre la validez de algunos de ellos usados actualmente y se trata de hacer algunas precisiones puntuales. La diversa terminología empleada en el manejo y difusión de la información complica el análisis de los mensajes informativos e impide establecer definiciones que tipifiquen la veracidad o la falsedad de los hechos.

Se reitera que la producción de mensajes informativos está supeditada e inmersa en el uso masivo de las TIC y posibilita, en muchas oportunidades, que estos mensajes sean irrelevantes desde la perspectiva de la veracidad, existiendo una especie de "inflación de la información" (aumento) que la degrada y la hace perder su valor constantemente (Doomen, 2009), este crecimiento desmesurado hace difícil la elección de información correcta. Por ejemplo, las redes sociales juegan un rol importante en la aparición de supuestos hechos carentes de veracidad y tienen un gran impacto por la magnitud de la escala en el que proliferan mensajes y por su horizontalidad.

En razón a lo anterior, el presente artículo tiene por objetivo precisar conceptos y delimitar la terminología utilizada (Disinformation y Misinformation, Posverdady Fake News), asimismo, demostrar que existen similitudes, diferencias y yuxtaposiciones entre estos términos utilizados en la literatura científica. Finalmente, se resaltará el papel del profesional de la información en este contexto.

\section{Metodología}

La hipótesis planteada para la presente investigación es la existencia de confusiones sobre el uso de conceptos pares como son Disinformation y Misinformation, con Posverdady Fake News, asimismo, ante la explosión informativa, existen similitudes, diferencias y yuxtaposiciones, porque se utilizan estos conceptos de diversa forma en las investigaciones sin llegar a concretar el uso correcto de cada uno.

La investigación fue de tipo básica, se realizó a través del enfoque cualitativo e interpretativo y se enmarcó en una investigación documental de carácter descriptiva, la cual determinó el procedimiento de selección, acceso y registro de la muestra documental (según interés de los investigadores). La técnica empleada ha sido la revisión documental y se construyó, como instrumento, una matriz para el registro en función a contenidos relacionados con disinformation, misinformation, posverdad y Fake News con los campos contenido, título, autor, año de publicación, sintesis, diferencias y similitudes. La búsqueda se realizó a través de Google Scholar. Una vez organizada la matriz se procedió a analizar parejas de términos. Esta revisión arrojó las similitudes, diferencias y yuxtaposiciones a efecto de identificar los términos más indicados para utilizarlos con propiedad según cada situación.

Se identificaron cuatro términos que funcionan en dos pares: disinformation y misinformation, posverdad y fake news que se utilizan indistintamente para conceptualizar uno a otro y se requiere hacer algunas precisiones para quienes estudien este fenómeno.

\section{Resultados}

En referencia a los resultados obtenidos, a través de la revisión documental, se estructuraron los términos en pareja que fueron analizados a lo largo de la investigación, que tienen relación de similitud y yuxtaposición, como son: Disinformation y Misinformation, posverdad y Fake News. Se prefiere utilizar algunos de los términos en el idioma original porque, en el español no hay una correspondencia exacta con la traducción o se usan indistintamente. 


\subsection{Disinformation y Misinformation}

En el vocabulario inglés existen dos términos para explicar la desinformación en dos sentidos. "Disinformation y misinformation (Mis/Dis) se contraponen, debilitan y afectan a la calidad de la información. Misinformation se puede definir simplemente como información falsa, errónea o engañosa" (Fetzer, 2004a: 231) aunque probablemente no hace daño, es inocua (Wardle y Derakhshan, 2017) y puede ser entendida como el desconocimiento de un tema o ignorancia, según la definición que da el Cambridge Dictionary (2018). "Disinformation, en particular, implica la difusión de información incompleta, inexacta o engañosa; tiene el objetivo, la meta o el fin de mentir deliberada o intencionalmente a otros sobre la verdad" (Fetzer, 2004b: 228); en resumen son dos formas de información inexacta.

Debido a que Mis/Dis se relacionan con discusiones y estudios de credibilidad, confiabilidad y engaño, a menudo es difícil determinar las motivaciones detrás de este intercambio de información errónea (Cooke, 2017). Mis/Dis son difícil de discernir en los medios en línea, donde hay gran cantidad de información (tanto precisa e imprecisa) y, a menudo, existe falta de respaldos visuales y auditivos que, en la vida real, podrían alertar al consumidor acerca de que algo es falso (Cooke, 2017).

\subsubsection{Misinformation}

La misinformation como información falsa debe tener contenido semántico y no representa los hechos como son; misinformation como información inexacta, la información incompleta puede ser perfectamente cierta pero la falta de información puede ser un tipo de inexactitud; misinformation como información engañosa, información que tiene la propensión a causar falsas creencias (Fallis, 2016). Se puede tipificar a los mitos informativos como una forma de difundir hechos irreales supeditados en el boca a boca y el rumor.

\subsubsection{Disinformation}

La desinformación al ser un acto de mentira es también intencional, porque se busca tergiversar un hecho y presentarlo como veraz. Asimismo, es un conjunto de engaños sistemáticos y organizados en un contexto en donde los medios de comunicación se hallan muy desarrollados (Durandin, 1995). La mayoría de las formas de desinformación, como las mentiras y algún tipo de propaganda (Brexit, Campaña electoral de Trump), son engañosas, porque la fuente pretende que así sea; sin embargo, otras formas de desinformación, como las teorías de conspiración y las falsas llamadas de alarma, son engañosas simplemente porque la fuente se beneficia sistemáticamente de ello (Fallis, 2015).

Hay tres requisitos para identificar una información confiable y que sea útil: que el conocimiento que el emisor desea comunicar sea lo más exacto posible; que el destinatario tenga una serie de conocimientos mínimos sobre lo que se quiere comunicar; que exista un código común entre las partes, es decir, un mismo lenguaje que sirva para analizar y describir el hecho o fenómeno (Durandin, 1995). Incluso desde la teoría de la información se intenta explicar la desinformación de forma sencilla como inyectar ruido en un subconjunto de la señal y reducir el contenido de información del mensaje o aumentando su entropía (caos), de esta forma no se destruye el mensaje original pero se intenta dañar un subconjunto de la información que es la más importante (Alexander y Smith, 2011).

La desinformación (disinformation) tiene tres características: la primera, la desinformación es información, específicamente un tipo de información que depende del análisis del contenido semántico o análisis representacional que se adopte, la información 
puede ser falsa pero también verdadera (en ambos casos es información). La segunda, la desinformación es información engañosa o confusa, es información que probablemente fomenta falsas creencias, genera daño y es peligrosa; va en contra de la calidad de la información. Finalmente, la desinformación es información no accidentalmente engañosa, es intencional, va desde las formas más inocuas como los errores honestos hasta la sátira sutil, el fin es simplemente engañar (Fallis, 2015), es defectuosa y peligrosa porque induce al error (Fallis, 2014).

\subsection{Posverdad y Fake News}

\subsubsection{Posverdad}

Los fenómenos de la comunicación e información en los últimos años han marcado la tendencia del discurso subjetivo o emotivo, así el Diccionario Oxford denominó a la palabra 'posverdad' (post-truth en inglés) como palabra del año en el 2016 y la define como un adjetivo relacionado a circunstancias en donde los hechos objetivos influyen menos en la formación de la opinión pública que aquellos que apelan a la emoción y a la creencia personal (Oxford-Dictionaries, 2016). Por tanto, según Oxford, la posverdad funciona cuando la gente prefiere hacer caso a sus creencias y emociones en lugar de la verdad objetiva.

El fenómeno de la posverdad es una corriente que se vale del subjetivismo por encima de la razón o los hechos. En el contexto global, este fenómeno se refiere a la relativización de la verdad, que no es sinónimo de mentira; hay una banalización de la objetividad de los datos o los hechos y evidencia una supremacía del discurso emotivo (Zarzalejos, 2017). Los hechos objetivos y lógicos pierden relevancia frente a un discurso que apela a la emotividad, creencias o sentimientos para ser aceptados por la sociedad, aunque siempre tiene algunos rasgos de verosimilitud.

Asimismo, para la Real Academia Española (RAE, 2016) la posverdad indica "toda información o aseveración que no se basa en hechos objetivos, sino que apela a las emociones, creencias o deseos del público". Cabe resaltar que el fenómeno de la posverdad no es un suceso reciente, siempre han existido las formas de ganar un debate o construir argumentos apelando a los discursos emotivos y a las creencias.

No obstante, el término 'posverdad' solo es un modismo o neologismo de esta época para referirse a este fenómeno; es una nueva forma de denominar a los discursos que no tienen sustento objetivo y se basan en la mentira. La posverdad es utilizada para debilitar o consolidar gobiernos y en diversas épocas han existido este tipo de manifestaciones que rehúyen a la verdad. Actualmente, vivimos en una era posterior a la verdad, una era en la que las audiencias son más susceptibles de creer en la información que apela a las emociones o las creencias personales existentes, en oposición a la búsqueda y la aceptación de la información considerada como factual u objetiva (Cooke, 2017).

La posverdad se ha ligado a la política y especialmente a las campañas electorales que han inundado de mentiras o de noticias que no se supeditan en hechos o la veracidad y no cumplen con los requisitos indispensables para el ejercicio de la libertad de información y fortalecimiento de la opinión pública. La estrecha relación entre el fenómeno de la posverdad y la política son evidentes, "Ciertamente, en la política la mentira o la media verdad siempre han sido recursos manejados con desenvoltura, pero, ahora, la respuesta al status quo político y económico ha introducido elementos sentimentales, emotivos, en sus mensajes falsos dotándolos de una fuerza arrasadora" (Zarzalejos, 2017: 12). 
La posverdad está relacionada también con la mentira, la desinformación, la banalización de la información y de los mensajes. Un discurso emotivo no es una mentira, pero muchas veces se puede entender como mentira, ya que no contiene argumentos objetivos y hechos verídicos que sean contrastados con la realidad, por tanto, cae en la categoría de desinformación y de mensajes falsos. Por ejemplo, "nuestras creencias no se basan en evidencias. No deberíamos ofendernos, ya que es una característica más de cómo somos los seres humanos, una que puede ayudarnos o perjudicarnos, según el caso" (Nogués, 2018).

La posverdad demuestra también una manipulación de la información y los hechos. Existe evidencia en los casos relacionados preferentemente con la política donde hay un intento por desinformar o decir 'medias verdades' para proteger determinados intereses. Se necesita conocer mejor los problemas actuales para "identificarlos, limitarlos o eliminarlos, de manera de pelear contra la posverdad casual y así, por extensión, poder hacerle frente también a la posverdad intencional" (Nogués, 2018).

La aparición de nuevos medios como Facebook o Twittery su inmediatez (compartir o retuitear), no permiten a las personas evaluar críticamente la información que se publica porque no hay un periodo de tiempo necesario para la reflexión o revisión de las fuentes. Además, los medios que nos proporcionan información son los referentes (familia, círculos de amistad, círculo profesional, partidos políticos, etc.), las instituciones (públicas, privadas, no gubernamentales) y los medios de comunicación. Estos agentes pueden estar relacionados a grupos de poder con ciertos intereses contrarios a la sociedad y, por ende, buscan las formas de desinformar, dar noticias falsas y banales. Por tanto, una mentira se construye a través de: "hacer creer que una cosa que existe, no existe; hacer creer que una cosa que no existe, existe; deformar una cosa que existe" (Durandin, 1983: 57).

\subsubsection{Fakenews (en el contexto de la posverdad)}

Para Filloux (2018) la pelea contra las fakenews está perdida porque existe una brecha cada vez mayor entre el volumen y las capacidades de procesamiento; en ese sentido, el progreso tecnológico está definitivamente en el lado de las fakenews. La información errónea continuará siendo alimentada por el acceso a una gran cantidad de recursos y gracias al hyper-targeting (focalización de mensajes), estos factores impiden corroborar las fuentes, autores, origen, medios de difusión, impacto y verosimilitud de la información.

Tandoc, Lim y Ling, identifican en la literatura científica seis tipos de fakenews como son la sátira (humor o exageración); parodia (difiere de la sátira en su uso de información no objetiva para inyectar humor y ridiculizar); fabricación (no tienen una base objetiva pero que se publican en el estilo de las noticias para parecer legítimos), manipulación (describe noticias visuales adulteradas); publicidad (destinada a vender o promocionar un producto, una compañía o una idea); propaganda (creadas por una entidad política para influir en las percepciones públicas) y establecen dimensiones en la revisión de definiciones de fakenews e identifican dos dominios calificados de alto a bajo. La factibilidad se establece en torno a los hechos tal como lo muestra la Tabla 1; por ejemplo, la sátira se basa en los hechos, pero tiene ciertas desviaciones, mientras que las parodias y las noticias fabricadas adoptan un amplio contexto social sobre el que se confunde con la ficción (Tandoc, Lim y Ling, 2018). 
Tabla 1.Tipología de definiciones de Fakenews

\begin{tabular}{ccc}
\cline { 2 - 3 } Nivel de factibilidad & \multicolumn{2}{c}{ Intención inmediata de engañar } \\
\cline { 2 - 3 } Alto & Alto & Bajo \\
\hline & $\begin{array}{c}\text { Publicidad nativa } \\
\text { Propaganda }\end{array}$ \\
Bajo & Manipulación & \\
& Fabricación & Parodia \\
\hline
\end{tabular}

En la actualidad, la emotividad, las noticias falsas y la desinformación se disfrazan de mensajes para ser difundidas entre las personas. En el ciberespacio hay una visibilidad que todos quieren alcanzar: cada uno persigue sus intereses, todos quieren ser visibles en las redes, todos quieren decir o escribir algo y muchas veces es un decir por decir.

Esta tendencia orientada al reforzamiento de las creencias es una amenaza para el análisis y argumentación crítica porque en las redes no se da importancia a los referentes (intelectuales o científicos), sino que se valora el contenido. Se observa que en la actualidad hay un aniquilamiento simbólico de las fuentes, ya sea por malas decisiones políticas o por intereses económicos: se cierran o abandonan bibliotecas, se persiguen periodistas disidentes, medios de comunicación promueven programas y contenidos banales porque se cree que todo entretenimiento es información, no hay políticas de protección al derecho a la información, hay un interés por el silencio y ocultamiento de la información.

Por consiguiente, la posverdad es un síntoma del estado actual de las sociedades o de la sociedad moderna, que tiene su causa en la globalización, crisis económica o crisis política. Otro efecto se puede notar en la opinión pública como referente objetivo, un espacio de debate entre lo verdadero y la falsedad, pero se ve banalizada por el crecimiento de la posverdad; dejan de funcionar los filtros, la corroboración de fuentes y la objetividad de la información.

Con la rapidez e inmediatez del internet, las fakenews y la desinformación se vuelven virales sin que sus contenidos sean examinados o confirmados. Sin embargo, si tal información se retrae, se refuta o se corrige, el daño ha sido hecho y la evidencia se archiva digitalmente y perpetuamente (Cooke, 2017: 211).

Hay muchas críticas a estos nuevos términos acuñados recientemente, aunque estos fenómenos han existido hace décadas. Habgood-Coote (2018) señala que estos neologismos (posverdad y fakenews) aluden a realidades distintas, muy habitualmente se da entre ellos una correlación temporal pero tienen tres problemas críticos: son lingüísticamente defectuosos, no tienen significados públicos estables; son innecesarios, porque no agregan ningún recurso descriptivo útil al idioma; y son políticamente problemáticos porque se utilizan para fines políticos y están estrechamente conectados a una ideología. La reacción natural a una palabra de moda mal definida es negarse a usarla.

\section{3. Ética de la información como respuesta}

Floridi (2006) define a la Infosfera como un complejo ambiente cuyo tránsito está constituido por todas las entidades informacionales (propiedades, interacciones y procesos) que circunda las sociedades; y sugiere que cada individuo o grupo de individuos existen como un nodo en una red de entradas y salidas de información, símbolos y lenguaje (Van der Veer Martens, 2015). Floridi establece el principio del valor moral de todas las entidades consideradas como objetos informacionales, es 
decir, desde la perspectiva de un principio irreductible del valor de la existencia (Brenner, 2010). Las TIC configuran nuevas formas y posibilidades de interacción entre personas y organizaciones y originan nuevas conectividades mediante el uso de estructuras informativas existentes en la Infosfera (Estrada-Cuzcano y SaavedraVásquez, 2018). Por tal razón, es imprescindible argüir respuestas éticas con respecto a la calidad de la información porque existe un "pánico moral informativo", relacionado con las tecnologías, que generan las desviaciones y distorsiones dentro de un grupo y establecen límites de aceptabilidad o resistencia que conllevan a una crisis comunicacional (Carlson, 2020). El proceso de comunicación requiere que la información sea verdadera, a su vez, compromete a sus difusores a ciertos principios y valores éticos, como la honestidad, la sinceridad, la veracidad, integridad y confiabilidad (Howlett Spence, 2010).

Estas nuevas formas y posibilidades hacen pensar en una ética de la información (EI por sus siglas en español) porque constantemente surgen problemas cuando algunas prácticas no están claramente definidas y es difícil establecer rangos, factores y condiciones informacionales dentro de una perspectiva correcta. Consecuentemente, el agente o mediador moral, cumple una labor fundamental en la gestión de los procesos de información como una cuestión crucial para agregar valor a la Infosfera (Hofkirchner, 2010). Además, los agentes morales contribuyen a una sociedad global consagrando una especie de justicia informacional de forma recíproca, participativa, distributiva y contributiva (Britz, 2008).

Para Floridi (2006) la ética de la información como propósito de estudio, por parte de los profesionales, se debe abordar desde varias perspectivas: la ética de la información como recurso (brecha digital, fiabilidad, confiabilidad y sobreinformación); la ética de la información como producto (responsabilidad, testimonio, propiedad, desinformación y plagio) y la ética de la información como objetivo (confidencialidad, intimidad y hackers).

Un aspecto transversal a tratar por la EI es la transparencia de la información que implica apoyarse en datos significativos, verídicos, comprensibles, accesibles y útiles (Turilli y Floridi, 2009) y permite el acceso en correspondencia con los principios éticos. Inclusive se podría establecer una ética de los datos (generadores de información) que se define como la rama de la ética que estudia y evalúa los problemas morales relacionados con los datos (incluida la generación, el registro, la curación, el procesamiento, la difusión, el intercambio y el uso), los algoritmos (incluida la inteligencia artificial, los agentes artificiales, el aprendizaje automático y los robots) y las prácticas correspondientes (incluidas las innovación, programación, piratería y códigos profesionales), para formular y apoyar soluciones moralmente buenas (por ejemplo, conductas o valores correctos).

Esto significa que los desafíos éticos planteados por la ciencia de datos se pueden mapear dentro del espacio conceptual delineado por tres ejes de investigación: la ética de los datos, la ética de los algoritmos y la ética de las prácticas (Floridi y Taddeo, 2016). Además, Floridi señala que cualquier tipo de destrucción o corrupción de entidades entendidas como objetos informacionales genera "desorden, degradación 0 aleatoriedad" (Hongladarom, 2008: 44).

Se puede argüir que en la EI todo objeto tiene valor informativo y toda destrucción de cualquier objeto informativo es inmoral e implica una pérdida de valor (Byron, 2010). De esta manera, propicia, entre otros, la accesibilidad, orden, exactitud, exhaustividad, transparencia, fiabilidad como propiedades aplicadas por los profesionales de la información elegidos como agentes morales; por tal razón, el desarrollo de la EI (como teoría ética) debe ser convincente para que los agentes la acepten o la sigan 
(Stahl, 2008: 98). Los agentes tienen una posición de privilegio que les permite conocer cómo crear, buscar, administrar, acceder, preservar y utilizar la información de manera efectiva que proporciona una forma de poder al profesional de la información (Carbo y Almagno, 2001: 511).

\section{Discusión}

Se comparan los resultados obtenidos en la revisión bibliográfica con el trabajo realizado por Del-Fresno-García (2019) donde menciona que como sociedad somos eficaces al abordar y superar debates filosóficos, pero no lo somos en protegernos de las consecuencias de los desórdenes informativos y las irracionalidades cognitivas. Cambiar la escala, alcance y velocidad e impacto de los desórdenes informativos, interrelacionados entre sí, en intersección con las nuevas tecnologías post internet está transformado la naturaleza misma de la comunicación interpersonal o colectiva, siendo muy significativa la dificultad para diferenciar las verdades fácticas de las ficciones.

El resultado es que las audiencias tienen confusión, distorsionan las percepciones y la comprensión de la realidad e impiden establecer criterios de valor para identificar la información fiable de la espuria o aquella sesgada de forma intencional. Es responsabilidad de los profesionales de la información asumir un papel protagónico en la ayuda y orientación a las personas proporcionando herramientas para comprender, discernir y usar la información que reciben a través de diferentes medios promoviendo condiciones para la evaluación crítica.

Se coincide con el trabajo realizado por Morales Campos (2018), quien concluye que los bibliotecarios y las instituciones que ofrecen información deben estar comprometidos con los hechos y la verdad. Asimismo, considera que cuando información falsa llega al usuario, le es muy difícil discriminarla. Las verificaciones necesarias, sumarán un tiempo extra a las búsquedas y trastocarán algunas cualidades que de origen nos ofrecían las TIC, como la rapidez, la oportunidad, la inmediatez, que permiten solucionar necesidades de información en el transporte público, en la oficina, en la casa, en la calle, en el café, es decir, habrá un impacto sobre las cualidades más significativas de estas tecnologías. Por lo tanto, además de las soluciones tecnológicas, debemos insistir en la educación de los usuarios y de los ciudadanos, para privilegiar los hechos, la información probada y la formación de valores éticos en la producción, difusión y uso de la información; es necesario formar a los usuarios para vivir en una sociedad plural, diversa, incluyente, al respetar los dichos y hechos del otro. La alfabetización informacional es un elemento clave en esta labor y contribuye a crear ciudadanos mejor informados.

\section{Conclusiones y recomendaciones}

1. El acceso desmesurado a grandes cantidades de datos e información, producto del desarrollo de las TIC, ha conllevado a la saturación y, por consiguiente, ha desplazado, en muchos casos, a la veracidad de la información y el tener una actitud crítica frente a la información que se recibe o se obtiene.

2. La veracidad de la información de las redes sociales, como las fuentes utilizadas, juegan un rol importante en la era digital, debido a que la información que ofrecen o que ponen a disposición del público, en diversas plataformas, responde usualmente intereses de diverso tipo.

3. Se considera a los conceptos misinformation y disinformation como términos más precisos que posverdad y fake news, neologismos surgidos recientemente. Misin- 
formation y disinformation son términos mejor conceptualizados y tienen un desarrollo teórico más sostenido y preciso.

4. La ética de la información y los profesionales de la información como agentes morales son una respuesta a la disinformation, misinformation, fakenews y posverdad y son imprescindibles para alcanzar una Infosfera transparente y accesible.

5. Los consumidores de información requieren desarrollar habilidades necesarias para ser críticos y creadores de información responsables. Asimismo, es imprescindible la alfabetización mediática para identificar mitos informativos y fakenews.

6. El papel de los profesionales de la información y de las bibliotecas es alfabetizar informacional y digitalmente a las personas para ayudarlos a estar en condiciones de poder evaluar la información, manejar fuentes de todo tipo y discernir críticamente entre la información falsa y verdadera.

\section{Agradecimientos}

Proyecto E19032051 financiado por el Vicerrectorado de Investigación y Posgrado de la Universidad Nacional Mayor de San Marcos. 


\section{Q Referencias bibliográficas}

"Alexander, James y Jonathan Smith. 2011. Disinformation: A taxonomy. En IEEE Security \& Privacy. Vol. 9, no.1, 58-63. <https://doi.org/10.1109/MSP.2010.141>

"Bradbury, Ray. 1953. Fahrenheit 451. New York: Ballantine Books.

» Brenner, Joseph E. 2010. A Logic of Ethical Information. En Knowledge, Technology E Policy. Vol. 23, no. 1-2, 109-133. <https://doi.org/10.1007/s12130-010-9099-3>

"Britz, Johannes J. 2008. Making the global information society good: A social justice perspective on the ethical dimensions of the global information society. En Journal of the American Society for Information Science and Technology. Vol. 59, no. 7, 1171-1183. <https://doi.org/10.1002/asi.20848>

»Byron, Michael. 2010. Floridi's Fourth Revolution and the Demise of Ethics. En Knowledge, Technology \& Policy. Vol. 23, no. 1-2, 135-147. <https://doi.org/10.1007/ s12130-010-9103-y>

"Cambridge Dictionary. 2018. Misinformation. <https://dictionary.cambridge. org/es/diccionario/ingles/misinformation> [Consulta: 11 diciembre 2018].

"Carbo, Toni y Stephen Almagno. 2001. Information ethics: The duty, privilege and challenge of educating information professionals. En Library Trends. Vol. 49, no. 3, 510-518. <http://hdl.handle.net/2142/8351> [Consulta: 23 setiembre 2018].

"Carlson, Matt. 2020. Fake news as an informational moral panic: the symbolic deviancy of social media during the 2016 US presidential election. En Information, Communication \& Society. Vol. 23, no. 3, 374-388. <https://doi.org/10.108o/1 369118X.2018.1505934>

»Cooke, Nicole A. 2017. Posttruth, Truthiness, and Alternative Facts: Information Behavior and Critical Information Consumption for a New Age. En The Library Quarterly. Vol. 87, no. 3, 211-221. <https://doi.org/10.1086/692298>

»Del-Fresno-García, Miguel. 2019. Desórdenes informativos: sobreexpuestos e infrainformados en la era de la posverdad. En El profesional de la información. Vol. 28, no. 3, 1-11. <https://doi.org/10.3145/epi.2019.may.02>

»Doomen, Jasper. 2009. Information Inflation. En Journal of Information Ethics. Vol. 18, no. 2, 27-37. <https://doi.org/10.3172/JIE.18.2.27>

»Durandin, Guy. 1983. La mentira en la propaganda política y en la publicidad. Barcelona: Paidós.

»Durandin, Guy. 1995. La información, la desinformación y la realidad. Barcelona: Paidós.

"Estrada-Cuzcano, Alonso y Valeria Saavedra-Vásquez. 2018. The Legal and Ethical Basis of Intellectual Freedom. En Journal of Information Ethics. Vol. 27, no. 1, 31-42.

» Fallis, Don. 2014. Epistemic Values and Disinformation. En Fairweather, Abrol, ed. Virtue Epistemology Naturalized: Bridges Between Virtue Epistemology and Philosophy of Science. Cham: Springer International Publishing. p. 159-179. <https:// doi.org/10.1007/978-3-319-04672-3_10>

" Fallis, Don. 2015. What Is Disinformation? En Library Trends. Vol. 63, no. 3, 401426. <https://doi.org/10.1353/lib.2015.0014> 
» Fallis, Don. 2016. Mis- and dis-information. En Floridi, Luciano, ed. The Routledge Handbook of Philosophy of Information. Oxfordshire: Routledge. p. 332-346. <https://doi.org/10.4324/9781315757544.ch27>

» Fetzer, James H. 2004a. Disinformation: The use of false information. En Minds and Machines. Vol. 14, no. 2, 231-240. <https://doi.org/10.1023/B:MIND.0000021683.28604.5b>

» Fetzer,James H.2004b.Information: Does ithave to betrue? En Mindsand Machines. Vol. 14, no. 2, 223-229. <https://doi.org/10.1023/B:MIND.0000021682.61365.56>

"Filloux, Frederic. 2018. Fighting fake news is a losing battle, but there are other ways to win the war. En Monday Note. 6 de agosto 2018. <https://mondaynote. com/fighting-fake-news-is-a-losing-battle-but-there-are-other-ways-to-winthe-war-1991ed877478> [Consulta: 12 enero 2019].

»Floridi, Luciano. 2006. Ética de la información: su naturaleza y alcance. En Isegoría. No. 34, 19-46. <https://doi.org/10.3989/isegoria.2006.i34.2>

» Floridi, Luciano y Mariarosaria Taddeo. 2016. What is data ethics? En Philosophical Transactions of the Royal Society A: Mathematical, Physical and Engineering Sciences. Vol. 374, no. 2083, 1-5. <https://doi.org/10.1098/rsta.2016.0360>

»Goldmann, Lucien. 1966. Importancia del concepto de conciencia posible para la comunicación. En Coloquios Royaumont. El concepto de la información en la ciencia contemporánea. México, D.F.: Siglo XXI. p. 31-41.

" Habgood-Coote, Joshua. 2018. Stop talking about fake news! En Inquiry. Vol. 62, no. 9-10, 1-33. <https://doi.org/10.1080/0020174X.2018.1508363>

" Hofkirchner, Wolfgang. 2010. How to Design the Infosphere: the Fourth Revolution, the Management of the Life Cycle of Information, and Information Ethics as a Macroethics. En Knowledge, Technology, \& Policy. Vol. 23, no. 1-2, 177-192. <http://dx.doi.org/10.1007/s12130-010-9108-6>

" Hongladarom, Soraj. 2008. Floridi and Spinoza on global information ethics. En Ethics and Information Technology. Vol. 10, no. 2-3, 175-187. <http://dx.doi. org/10.1007/s10676-008-9164-8>

»Howlett Spence, Edward. 2010. The normative structure of information and its communication. En Journal of Information, Communication and Ethics in Society. Vol. 8, no. 2, 150-163. <http://dx.doi.org/10.1108/14779961011040569>

" Morales Campos, Estela. 2018. Desinformación en la Sociedad de la Información y el Conocimiento. En Morales Campos, Estela, ed. La posverdad y las noticias falsas: el uso ético de la información. México, D.F.: UNAM, Instituto de Investigaciones Bibliotecológicas y de la Información.

"Nogués, Guadalupe. 2018. Creencias y emociones ¿Cómo nos afectan las emociones a la hora de interpretar los hechos? En Nogués, Guadalupe, ed. Pensar con Otros: Una guía de supervivencia en tiempos de posverdad. Buenos Aires: El Gato y La Caja. <https://elgatoylacaja.com.ar/pensar-con-otros/capitulo-5/> [Consulta: 15 marzo 2020].

》 Orwell, George. 2016. 1984. México, D.F.: Tomo.

» Oxford-Dictionaries. 2016. Oxford Dictionaries Word of the Year 2016 is... <https://en.oxforddictionaries.com/word-of-the-year/word-of-the-year-2016> [Consulta: 22 diciembre 2018].

» RAE. 2016. Diccionario de la lengua española. 23a. <http://dle.rae.es/> [Consulta: 22 diciembre 2018]. 
»Ranganathan, Shiyali Ramamrita. 1931. The five laws of library science. Madras: Madras Library Association; Londres: Edward Goldston.

"Stahl, Bernd Carsten. 2008. Discourses on information ethics: The claim to universality. En Ethics and Information Technology. Vol. 10, no. 2-3, 97-108. <https:// doi.org/10.1007/s10676-008-9171-9>

»Tandoc, Edson C., Zheng Wei Lim y Richard Ling. 2018. Defining "Fake News”. En Digital Journalism. Vol. 6, no. 2, 137-153. <https://doi.or g/10.108o/21670811.2017.1360143>

»Turilli, Matteo y Luciano Floridi. 2009. The ethics of information transparency. En Ethics and Information Technology. Vol. 11, no. 2, 105-112. <https://doi. org/10.1007/s10676-009-9187-9>

"Van der Veer Martens, Betsy. 2015. An Illustrated Introduction to the Infosphere. En Library Trends. Vol. 63, no. 3, 317-361. <https://doi.org/110.1353/lib.2015.0006>

"Wardle, Claire y Hossein Derakhshan. 2017. Information Disorder: Toward an interdisciplinary framework for research and policy making. Strasbourg: Council of Europe. <https://rm.coe.int/information-disorder-toward-an-interdisciplinary-framework-for-researc/168076277c> [Consulta: 17 noviembre 2018].

»Zarzalejos, José Antonio. 2017. Comunicación, periodismo y ‘fact-checking’. En Uno. No. 27, 11-13. <http://www.revista-uno.com/wp-content/uploads/2017/o3/ UNO_27.pdf> [Consulta: 29 noviembre 2018]. 